\title{
CLOSING THE GAP: A RESEARCH AGENDA FOR THE STUDY OF HEALTH NEEDS AMONG AMERICAN INDIAN/NATIVE HAWAIIAN TRANSGENDER INDIVIDUALS
}

\author{
Irene $S$ Vernon \\ Colorado State University \\ Trudie Jackson \\ University of Arizona
}

Despite treaty agreements and Native focused health efforts, health disparities among American Indians and Native Hawaiians (AI/NH) have persisted for decades as federal, state, and tribal efforts to address them have waxed and waned. These disparities are even more pronounced among lesbian, gay, bisexual, and transgender (LGBT) individuals. The National Institutes of Health (NIH) has established that LGBT people experience, over a lifetime, an array of health issues such as victimization, violence, anxiety disorders, substance abuse, HIV, stigma, and discrimination and have recommended that an agenda be enacted that will "assist NIH in enhancing its research efforts in the area of LGBT health ... and advance understanding of LGBT health" (National Institute of Medicine [NIM], 2011, p. 6). The need to understand the variation found among LGBT people is vital and many have made a call to address this lack of information and to help close the health disparity gap (Lombardi, 2010; NIM, 2011). In an effort to add to the literature and, more importantly, to provide information on how to better serve and improve the health of American Indian and Native Hawaiian transgender people, this study identified key health research needs.

The term transgender is complex and has a number of meanings but generally it describes "people who have gender identities, expressions, or behaviors not traditionally associated with their birth sex" (Mayer et al, 2008, p. 990) and may include cross-dressers, transvestites, transgenderists, drag queens/kings and other gender variant identities. Among AI/ $\mathrm{NH}$ people there are regionally specific terms for transgender that may include cultural terms such as mahu, mahuwahine, mahukane, winkte, heyoka, nádleehí, ditbah, and the contemporary term two-spirit (Roscoe, 1998). It is important to note that the transgender term is evolving and varies by region and individual. 
To obtain basic information on the current health status of $\mathrm{AI} / \mathrm{NH}$ transgender individuals an exploration of $\mathrm{AI} / \mathrm{NH}$ and transgender populations had to be explored independently, given the dearth of scholarship specifically focused on $\mathrm{AI} / \mathrm{NH}$ transgender individuals.

In the United States the collection of health data on racial/ethnic minorities and sexual minorities has been fraught with problems. For AIs, the collection, analysis, interpretation and dissemination of their health data have led to many questions. Questions about AI health data have arisen due to the findings of racial and/or ethnic misclassification, poor data management, and inept coordination of health data between states and tribes (Vernon, 2012, p. 173, 174). As well, problems with the collection of LGBT health data have a long history of challenges and includes concerns such as: “(1) operationally defining and measuring sexual orientation and gender identity, (2) overcoming the reluctance of some LGBT individuals to identify themselves to researchers, and (3) obtaining high-quality samples of relatively small populations" (NIM, 2011, p. 89). One of the intentions of the Affordable Care Act, however, is the mission that health data collection will be improved and better coordinated. The Act requires data collection standards for key demographic variables such as race, ethnicity, sex, language and disability status (Dorsey et al., 2014). The United States Department of Health and Human Services' (HHS) decision to limit its question on sex/gender to merely male and female categories on its National Health Survey is problematic, and though the HHS survey has included questions on sexual orientation in their National Health Interview Survey (Dorsey et al., 2014, p. 130), categorization should be further expanded.

Although limited health literature exists on transgender populations a recent article on LGBT health disparities and the Health Equity Promotion Model, by Fredriksen-Goldsen et al. (2014), reports that in two of the most current studies with large national samples of transgender individuals the "rates of depression, anxiety, and overall psychological distress were disproportionately higher for this population than for nontransgender women and men" (p. 654). Another national study that focused on transgender people and discrimination found that " $41 \%$ of respondents reported attempting suicide compared to $1.6 \%$ of the general population, $19 \%$ were refused medical care due to their transgender status, $28 \%$ postponed medical care due to discrimination or inability to afford it (48\%), and those who expressed their transgender identity in grades K-12 reported alarming rates of harassment (78\%), physical assault (35\%) and sexual violence (12\%) (Grant, Mottet, Tanis, 2011, p 2, 3). Other smaller studies have focused on HIV, substance abuse, violence, mental health, and hormone usage (Feldman \& Safer, 2009; Operario, 2011; Operario \& Nemoto, 2005; Testa et al, 2012; Shipherd, et al, 
2010.). The National Institute of Medicine's book The Health of Lesbian, Gay, Bisexual, and Transgender People (2011), a leading text, confirms that over their lifetime LGBT individuals encounter a number of health issues including HIV, suicide, violence, stigma, discrimination, substance abuse, homelessness, and mental health disorders.

Literature on $\mathrm{AI} / \mathrm{NH}$ trans gender people is extremely limited but it has been well established that they have a high prevalence of HIV, high rates of abuse and trauma, are medically underserved, and experience discrimination, violence, and traumas that are linked to adverse health outcomes. Specific to $\mathrm{NH}$ transgender populations there are only two prominent articles in the field (Ellingson \& Odo, 2008; Odo \& Hawelu, 2001). Odo \& Hawelu (2001) found that compared to the general NH population; $\mathrm{NH}$ transgender individuals had substantially higher rates of HIV, smoking cigarettes, drug use, and no medical insurance, and were more exposed to violence (p. 332). A later study noted that $\mathrm{NH}$ transgender individuals were at heightened risk of HIV infection from engaging in unprotected receptive anal intercourse, sex work, and having sex while high, and illicit substance use (Ellingson \& Odo, 2008, p. 562).

Literature on AI transgender health is equally as limited as $\mathrm{NH}$. When searching for AI transgender health only one article appears that is focused on sexual partner concurrency and sexual risk among GLBT AI/ Alaska Natives. The findings of this article recommend more attention paid to this population given its high rates of HIV and sexual partner concurrency (Cassels, Pearson, Walters, Simoni, \& Morris, 2010, p. 277). A few more articles can be found when searching "two-spirit," which is a contemporary term, adopted in 1990 at the International Native Gay \& Lesbian Gathering in Canada, used to describe Native American, Alaska Native, lesbian, gay, bisexual, and transgender (LGBT) individuals. This term however is not used by all Native people and will have different meanings in different communities.

An early study of two-spirits found that compared to heterosexuals they reported twice the rate of childhood exposure to physical abuse from their caretakers, significantly more "native specific historical trauma occurring in the lives of their parents, grandparents, and great grandparents," and "significantly higher rates of lifetime illicit drug use" (Balsam, Bu, Fieland, Simoni, \& Walters, 2004, p. 297). Other studies have found that their higher reports of discrimination were associated with significantly greater odds of reporting physical pain and impairment (Chae \& Walters, 2009, p. S149); greater victimization with more lifetime HIV risk behaviors and risker sexual partners than their heterosexual counterparts (Simoni, Walters, Balsam, \& Meyers, 2006, p. 3); and high alcohol misuse (Yuan, Duran, Walters, Pearson, \& Evans-Campbell, 2014, p. 10470). Given this limitation of the research an exploration 
of health perception within the $\mathrm{AI} / \mathrm{NH}$ and transgender population independently provides a more complete picture of their health status and health needs.

Currently, AIs/NHs suffer disproportionately from a number of diseases and illnesses (i.e. diabetes, mental health disorders, cardiovascular disease, etc.), and health disparities that have persisted for over 500 years (Jones, 2006; U.S. Commission on Civil Rights, 2004). Likewise, over their life span, transgender people also report higher risk for suicidal ideation, high rates of smoking and substance abuse, and frequent experience of violence, discrimination and stigma (NIM, 2011, p. 4, 5). It is significant that the poor health outcomes for $\mathrm{AI} / \mathrm{NH}$ and transgender populations are rooted in histories of oppression, discrimination, and stigma (Jones, 2006; NIM, 2011, p. 32-48; U.S. Commission on Civil Rights, 2004) and it is imperative that this fact be understood and acknowledged in order to develop effective health care services for $\mathrm{AI} / \mathrm{NH}$ transgender individuals. When the independent health data on $\mathrm{AI} / \mathrm{NH}$ and transgender individuals are combined, it is notably relevant that $\mathrm{AI} / \mathrm{NH}$ transgender people encounter serious health challenges that have been overlooked or ignored by health and government officials as well as the research community. Certainly, there is a serious need for more scholarship and better-informed and comprehensive services.

This study explores the health and research needs of AI/NH individuals using their voices to help identify deprivations and deficiencies in their access to quality mental and physical healthcare and in existent research programs in this area. Given the lack of theory generated in this health domain, a grounded approach is used here.

\section{MethodS}

Background. This project began by the principal investigator being approached by a nádleehí ${ }^{1}$ who works in the field of HIV/AIDS suggesting that the principle investigator examine the research needs of AI transgender individuals given their high rates of health disparities. The principal investigator has worked in the field of AIs and HIV/AIDS for several decades but not specifically with the transgender population. To familiarize herself with their health needs and issues the principle investigator had an informal meeting with seven AI transgender individuals in Los Angeles, CA. From that meeting she formulated an exploratory project on the research needs of $\mathrm{AI} / \mathrm{NH}$ transgender individuals utilizing a

1 Nádleehí is a Navajo term that describe a biological born male that shows feminine characteristics such as dressing up in female clothing, wearing make up, assisting with ceremonials by taking leads as head cook and helping dress ceremonial patient. In Navajo Creation Stories, the nádleehí were once revered among the Navajo people by showing feminine/masculine characteristics in taking on various roles as needed within communities and households. 
qualitative CBPR process. Following the precepts of CBPR it was important to recognize and value health research priorities identified by the community understudy.

Participants and Recruitment. Of the forty-two participants in this project all self-identified as transgender and AI or NH. Forty-one identified as male to female and one identified as female to male. This study is somewhat national in scope in that qualitative data were collected in Los Angeles, CA, Chicago, IL, Albuquerque, NM, Phoenix, AZ, and Honolulu, HI, between 2011 and 2012. The original meeting that took place in Los Angeles, CA and was coordinated through the Red Circle Project, at AIDS Project Los Angeles, that provides services to Native Gay/TwoSpirit men and Native male to female transgender individuals. At that meeting the principle investigator met with seven AI transgender people to elicit thoughts and ideas of a project to gather information on the health and research needs of AI transgender individuals.

From the Los Angeles meeting a project was developed to examine both the current health milieu and the health needs for AI/NH transgender populations. Four two and one half hour focus groups were conducted. One was conducted at the United States Conference on AIDS, two at LGBT community centers, and another at an HIV/AIDS organization. The coordinators who helped with the project chose the focus group sites based on their networks and connections to $\mathrm{AI} / \mathrm{NH}$ transgender populations. To keep the research participant names and identifying information confidential, Colorado State University's institutional review board (IRB) approved the use of cover letters instead of the use of a signed consent form. Once participants indicated a willingness to participate a cover letter email was sent to them by the principal investigator. The letter informed participants of the confidentiality, the risks, the benefits, and highlighted the overall goals of the project: (1) to develop a research agenda/proposal and (2) to develop articles based on the identified needs of $\mathrm{AI} / \mathrm{NH}$ transgender populations. It also provided the following: title of the project, principal investigator contact information, procedures, eligibility criteria, compensation, and voluntary participation clause. The same letter was given to participants again at the beginning of the focus group. The principle investigator then reviewed the letter and reiterated to the participants their right to withdraw their consent and cease participation at any time without penalty or loss of benefits to which they were otherwise entitled.

For recruitment one nádleehí and three $\mathrm{AI} / \mathrm{NH}$ transgender individuals were hired to recruit participants and assist in the coordination of activities. Coordinators were paid to locate focus group facilities, determine the appropriate compensation for participants, and, in some cases, determine the culturally appropriate food to be provided to ensure partic- 
ipation. Incentives such as gifts, food, and money are commonly used and recommended in research with AI populations (Strickland, 1999) and in this project all focus groups ended with an informal meal for participants. The principal investigator employed a note taker or facilitator at each site. All note takers or facilitators, with the exception of one, were AI or NH and had established experience with focus group activities. All were oriented to both the confidentiality issues and the goals of this project prior to the focus group. All focus groups were conducted in English.

Sampling and Data Collection. For this hard-to-reach population, snowball sampling was used knowing that a "degree of trust is needed to initiate study subjects' recruiting" (Shaghaghi, Bhopat, \& Sheikh, 2011, p. 4). AI/NH gay or transgender coordinators helped recruit participants for the Structuring an American Indian/Native Hawaiian Transgender Research Project. All coordinators worked in the field of health and/or in transgender communities. Criteria for participation in the project included being (1) over the age of 18 and (2) self-identified as an AI/NH transgender person. The principal investigator or the coordinator contacted potential participants by phone, email, or in person and described the project in an effort to determine their willingness to participate and ensure confidentiality. When a potential participant indicated a willingness to engage in the focus group, the principal investigator emailed a cover letter to them.

Data Collection. Qualitative inquiry was selected for this project because it allows for "a more detailed account of individuals' experiences as members of LGBT populations" (NIM, 2011, p. 120). Focus groups were conducted in an effort to better understand the experiences of the health issues facing $\mathrm{AI} / \mathrm{NH}$ transgender persons building on the interaction within the group. In conjunction with the qualitative method, the process of community based participatory research (CBPR) provided the foundation to collection information that may improve health equity, give a voice to $\mathrm{AI} / \mathrm{NH}$ trans gender individuals, and most importantly, create trust between researcher and $\mathrm{AI} / \mathrm{NHs}$ (Wallerstein \& Duran, 2010). Mistrust between AIs and researchers has a long and valid history based on unethical encounters. The implementation of CBPR has been used to break this cycle given its collaborative approach. It equitably involves and honors all partners, allows for the building of community capacity and trust, and ensures that the research benefits the participants (Pachecho et al., 2013; Thomas, Rosa, Forechimes, \& Donovan, 2011).

Instrumentation. Focus group questions were developed by the principal investigator and shared with three AI LGBT individuals. Utilizing that input, an additional question (RQ6) was added to further expand on the definition of "identity." The facilitator schedule included the following research questions: 
RQ1. What do you think are the most significant health related issues for transgenders?

RQ2. What do you think are the most significant barriers to health care for transgenders?

RQ3. What do you think are the strengths and resiliencies of transgenders?

RQ4. Tell me the way in which the care environment could be improved for transgenders?

RQ5. What would you recommend that a health research on transgenders focus on?

RQ6. What are your thoughts on the term Two-Spirit or your own tribal word?

RQ7. Is there anything you would like to add?

Data Analysis. The approach to the data analysis utilized a combination of grounded theory analysis and the principles of CBPR. Data for analysis included transcripts, field notes, and memos. Utilizing a grounded theory approach the principal investigator allowed the themes and theory to grow out of the data rather than analyzing the data with a theory already in place (Charmaz, 2006). The principal investigator engaged in four levels of analysis beginning with initial and open coding, labeling each line, focused coding and category development, axial and thematic coding. The resulting theories emerged from the categories and themes. The principal investigator utilized memos throughout the process to provide a record of her analytical process, thoughts, feelings, and insights into the project. Another researcher familiar with grounded theory and AI health also coded the data utilizing grounded theory. Both researchers approached the data inductively with no priority hypotheses and throughout the process made constant and consistent comparisons. Finally, the principal investigator compared and contrasted the data analyses by further refining the theories and major themes and then shared the results with a transgender community advisory board (CAB) that consisted of three AI transgender individuals. Given the lack of funding for this project, member checks were not done with all participants but instead only with $\mathrm{CAB}$ members. The $\mathrm{CAB}$ members reflected the community, "sharing a common interest, identity, experiences, history, language and culture" (Newman, et al., 2011, p. 1) and were drawn from project participants. CABs have been found to be an important factor in discussions of community perceptions and priorities in the development of a research agenda/process. In this project the advisory board members were invited to review the focus group transcripts and the investigator's findings and share their insights and thoughts on the data. The members assisted with interpretation of the data and related the results to larger cultural and social contexts. All advisory board members were compen- 
sated for their contributions of time and expertise. Follow-up questions were asked as well of several other focus group participants about the composition of a proposed "transgender health care package," and they too were compensated for their time.

\section{RESULTS}

Two main themes emerged from this project, healthcare and resiliency, and within each were a number of subcategories.

\section{Healthcare}

In this study most of the participants reported receiving poor quality healthcare stemming from health professionals' attitudes and treatment, quality care unique to their transgender status and age, along with inaccessibility to hormones.

Health Professionals' Attitudes and Treatment. A consistent comment from participants was that they did not receive quality care due to treatment by the health professionals. As one participant commented, they need to "treat us as human beings, not like a freak." Participants expressed that health professionals were insensitive and rude to their identity through the use of their birth names and inappropriate pronouns. They shared, "the nurse practitioner was unable to understand or accept [my] transgender identity" and "she called me by my other name which I do not prefer . . . some do it intentionally just to be mean." Participants described various encounters with health care workers who stared and gawked at them, "sometimes nurses look at me differently," "when one sees a male name they are like wow and then like I'll see people popping into the room just to look, like am I supposed to have another leg or something," and "they came in to gawk at me in the medical room." On a positive side, there were some health professionals who they indicated helped them get what they needed by "adjusting their hours for them," and helping them complete insurance forms and if needed, "doing it under the table because he understood that we needed certain things for our hormone regimen."

Access to Care that is Unique to Their Transgender Status. Given their transgender status many participants noted the lack of access to transgender specific health needs including qualified medical professionals, transitional care/services, and specific concerns related to hormones. All participants noted the difficulty of finding a doctor knowledgeable about transgender issues and how they had to do "lots of doctor shopping, at least six" because "there is not much to pick from." Many remarked how doctors ignored their transgender status and either focused on male or female issues independently not providing the holistic care/ services they needed and desired. They said, "they are going to do a 
physical for male instead of like a woman because I have breasts, I want to talk about mammogram, I want to talk about breast cancer, I want to talk about upper body women's issues." Another spoke, "for me I still have the parts I was born with, there is still a concern about prostate cancer and even though I am a woman, I neglect to get health care for checkup for male genitalia."

Poor Elder Transgender Care. Older participants spoke of their unique needs considering their many years of medical treatment and growing age. They not only talked about lack of services but of their mental health and of loneliness and invisibility. "We are suffering" was the statement of one participant who reflected many voices. "When looking at other women with full surgery you do not see them living past 60 years," "Where is our older generation?" and "When you feel that no one cares then you do not care for yourself." Participants noted how their loneliness and invisibility was not only created by society at large but within their own group, suggesting that the older they became the less support they had from other $\mathrm{AI} / \mathrm{NH}$ transgender individuals. Families also rejected them, "One gets married and has children, then later on in life decides this is who I am and transitions. Then his or her own children disown them. . . they are older and they worked all their life supporting this family unit. . . in my culture the young take care of the old. But, they are stuck by themselves, they are not invited to family functions they are. . very lonely." Elder participants expressed additional needs as they aged and how "because of our needs it becomes hard to be a woman, many of us revert to being a man again." Many mentioned the need for medical professionals to address their unique transgender status along with their aging needs.

Hormones. Many participants sought hormone replacement therapy (HRT) to align their appearance with their gender identity and this study found HRT to be a dominant subcategory within transgender specific needs. Participants obtained hormones from traditional and nontraditional sources. Individuals with "good" insurance accessed hormones through their physicians, medical centers, health clinics and Indian Health Service. For participants who had no insurance or had insufficient resources to purchase hormones found cheaper (and riskier) venues such as acquiring them from Mexico, Thailand, Canada, the Internet, friends, medical office staff, and the black market. Some participants reported that they had engaged in sex work and stolen doctor's prescription pads to obtain hormones. Participants, and at times even the doctors, "worked" the health care system to obtain hormones. From the statements documented, participants were savvy about ways to get what they needed. They reported that they had lied to their doctors to get a full hormone shot while some doctors gave them shots "under the table" or 
charged them less. Obtaining services/care that one needed could vary by costs and by the relationships between health care providers and the transgender individuals. For example, to address the problem associated with the monitoring of hormone treatment, those who were perceived as caring doctors found ways to charge insurance companies, generally categorizing it as another procedure. One participant shared that her doctor "did not want testing or lab work to hinder her from accessing treatment, therefore, if she was unable to do the lab work, he would still give her treatment but require that she return for monitoring." Another doctor also worked with insurance claims by filing them in a way to provide what the patient needed, "if you have insurance he doesn't say I am going to give this male patient a female hormone replacement therapy. He just says 'I am testing them for whatever' but what he is looking for are the levels in the liver. He does the whole thing so he can charge the insurance for the lab work if you have insurance. . if he says that he is doing it for hormones it is not covered by the insurance."

Participants were aware of the possible harmful effects of long-term use of hormones as well as the lack of medical training on the use and management of hormones. They willingly shared their experiences, "I knew someone who took those [black market hormones] and they made her breasts grow really weird," "I have been taking them since I was 19 years old. We all get them but who maintains them? We are putting ourselves at risk. I once shot into a muscle and one time I shot myself and could not walk for days." Concerns expressed over the risk of long-term use were plentiful and participants noted, "there are risks and side effects of hormones, like cancer risk, thyroid, and liver enzymes" and "Hormones change you." Many noticed the lack of hormone management, "Some girls take them like skittles" and they self-medicate not knowing how much to take. Many individuals reported that they saw changes when they stopped abruptly so they tried to self-manage, "you learn to be able to judge your levels of hormones."

Persistent through all focus groups was a concern related to the purchase and use of "bad hormones/street drugs." Participants noted how most did not know what they were buying and at times it was a combination of drugs and hormones. They commented, "black market hormones are putting me at risk," "many hormones are not approved, it is horse piss," and "some have died." One particularly telling comment regarding risk was made related to the quality of the black market hormones, "When I was living in L.A. a girl was selling vials. I was thinking of using it but then found that there $\mathrm{w}$ as no expiration date on it. Then it hit me, how long have those been out? It scared me. If I didn't think of that I probably would have taken it. I even got the syringe for it too." Even though many know the risk of non-prescribed hormones they seek them 
out anyway because as many spoke, it "fulfilled who I am, it completes me."

\section{Resiliency}

Resiliency, the ability to bounce back, in the face of adversity, was a major theme that included the subtopics of how it was built through their lived experiences, culture, and history.

Lived Experiences. Participants in all sites repeatedly expressed how their life experiences contributed to their resiliencies and enhanced their ability to bounce back. They stated that the neglect and ostracism that they experienced built strength. "As a whole you have to be pretty resilient to be. When you are in the world and your parents, family, church, and community are telling you 'what the hell are you wearing girls clothes for? Are you fucking crazy? What is wrong with you?' You have to be pretty strong to defy the odds, to defy your family and society." Another participant expressed similar sentiments, "I think the strength is because we have so much against us and you have to be strong or else. You have no choice. Like myself, I have no family, so I have to stand on my own two feet and fight for myself. And I think that is why trans people are so strong." Participants indicated that it was the daily pressures that helped many build fortitude. "Our strength comes from what we have to deal with on a daily basis. What makes us stronger is a lot of that experience, of that negativity that we grew up with, we had over and over again, made us stronger" and "how much we go through on an everyday basis. Life is hard but we always find a way to get through it, each day - inner strength."

In addition to lived experiences, the transgender participants spoke of their culture and histories as contributing to their resiliencies.

Culture. Many participants found "culture to be a big strength" and were able to distinguish key core cultural elements that helped build their tenacity. A number of AI/NH transgender people found meaning and purpose in their lives by helping and supporting others. Participants shared that their community was suffering and this became the impetus to help others. They spoke of advocacy and unity, "we need to stand together, that is the only way it will change." They stated that being or having role models/mentors were important to help guide, lead, and build a sense of hope and "to maneuver my way to get things done." Depending on their needs, participants had a variety of mentors/role models. The role models/mentors were American Indians, Native Hawaiians, non-Natives, Native spiritual leaders, Christians, men, transgender individuals, queens, performers, and family members. Participants commented that helping others was a core cultural value that they were taught and followed. Participants spoke, "Our spirit is that I would not let them be on 
the streets although much has been lost, it is in our life to help take care of kids and be peacemakers, this is cultural," "we are caretakers of babies and elders and still this continues to be our roles today" and "in my culture, the young take care of the old."

$\mathrm{AI} / \mathrm{NH}$ transgender individuals also spoke of how "Culture gives you an answer as to why there is a place for you." Participants further commented "in Native Hawaiian and Native American culture there is a place there for people like me" and "Our cultures and our traditions, what we heard in our stories in class made us stronger and for me, I can say that learning my culture and my traditions ... has helped me a lot." Another participant spoke of how they "need that place in society ... in my culture" to survive. Culture was also seen as a means to facilitate transition, "culture ... this is the strength behind those who are transitioning." It was noted, "Native Americans are more supported by their culture and from the time they are young they recognize the difference in someone who is going to transition . . . they recognize it and value it."

History. Historically, AI/NH histories included the acceptance of alternative genders and participants in all focus groups indicated that they wanted to preserve that history, learn more about it, and teach it to others. One participant spoke of their tribal traditions and how "Knowing the role of nádleehí can save the culture and increase self-esteem." A $\mathrm{NH}$ noted that in "Hawaiian culture, we are caretakers of babies and elder and still this continues to be our roles today, I would be a caretaker of my mother, the strength of our mahana." They expressed that they wanted younger transgender individuals to know that "back in the old days gender was not determined by what was between your legs it was determined by the chores that you do and the role that you played." AV/ $\mathrm{NH}$ individuals spoke of the cultural importance of family and, although not all Native families were accepting, participants expressed that they felt they were given a place and relied upon as a source of advice for others. They were also thankful that the elders who knew their history would "speak to extended family who were not immediately accepting" of them. History provided many of the participants with a sense of belonging and grounding. As one participate spoke, "remembering our history is important to make it a better day today."

\section{Discussion}

While it is true that many of the health research needs found in this study are similar in nature to the needs of other transgender people, they also reinforce research areas that the Institute of Medicine consider "essential for building a solid evidence base in LGBT health" (NIM, 2011, p. 7). NIM found that "LGBT individuals face barriers to equitable health care that can have profound impact on their overall wellbeing" 
and strongly encourage more research to "provide a solid basis from which to address these inequities" (p. 297). The data in this study certainly support the concept that there are unique research areas that need further exploration if the United States is to close the health care gap found among $\mathrm{AI} / \mathrm{NH}$ transgender people.

Current research has established that LGBT individuals will not seek care or disclose personal information when receiving care because of discrimination, stigma, and lack of provider knowledge (Clark, Landers, Linde, \& Sperber, 2001; Grant et al., 2011). Discriminatory actions by medical providers are found to be commonplace for transgender individuals who are denied equal treatment and sometimes denied services completely (Grant et al., p. 5). The National Transgender Discrimination Survey Report on Health and Health Care (2010) noted that many transgender individuals postponed their care because they could not afford it while others did so because of the "discrimination and disrespect from providers" (p. 7). The current study corroborates that research.

It is critical that health providers and agencies begin to develop and adhere to better-informed standards of care and related services. Participants spoke often of negative and discriminatory attitudes exhibited by health professionals, the lack of access and training, and the extent to which these factors have been found to "contribute to disparities in LGBT health" (Potter, Goldhammer, \& Makadon, 2008, p. 3). In another qualitative study, physicians spoke about the lack of transgender specific medical knowledge, lack of available resources, inadequate referral networks, and the challenges that arise from a health system that is "two-gendered" (Snelgrove, Jasudavisius, Rowe, Head, \& Bauer, 2012). In many cases the quality care of $\mathrm{AI} / \mathrm{NH}$ transgender people by medical professionals depended upon their relationship with individual transgender individuals and this should not be the case. The quality of care should be based upon the training of the medical professionals. Many have noted the need for more training and education. The need is further expressed through the limited and varied quality of instruction found in medical curricula. In a recent study it was found that "the median time reported dedicated to LGBT-related content in medical school in 20092010 was 5 hours" (Obedin-Maliver et al, 2011, p. 976). Several of the $\mathrm{AI} / \mathrm{NH}$ transgender individuals who participated in this study, worked in the health field and utilizing their knowledge to guide the curricula in these trainings would be advantageous given their knowledge of the health field, transgender issues, and $\mathrm{AI} / \mathrm{NH}$ cultural issues.

The Redfern and Sinclair article (2014) provided sound recommendations for improving health care encounters, suggesting changes be made to the office environment that includes gender-neutral bathrooms, the use of correct pronouns in all medical documentation, personnel 
training be increased, and that a network of health providers, that provide transgender health care and services, be established for advice and referrals. These suggestions should be extended to the Indian Health Service too. Attesting to the discrimination AIs encounter in the health care system, the National Center for Transgender Equity and National Gay and Lesbian Task Force indicated in their discrimination survey that $34 \%$ of AIs refused medical care due to bias and $64 \%$ postponed care when they were sick or injured due to fear of discrimination (Harrison-Quitana, Fitzgerald, \& Grant, 2012).

Data themes related to health challenges from older transgender individuals were often alarming and consistently demonstrated how the combined stigma of being elderly and transgender is fraught with serious health impact. Adding to this is the powerful impact of internalized oppression, given the findings in this study that elderly felt marginalized even in their own communities. The facts learned related to accessing health for older participants in this study was profound but not unlike the findings among many aging transgender individuals who have expressed fear of accessing health service due to discrimination and stigma (Fredriksen-Goldsen et al., 2014).

Given the many identities found among $\mathrm{AI} / \mathrm{NH}$ people there is a need for a more nuanced research projects among them to delve deeply into their needs. An intersectional research approach has been promoted by the NIM $(295,296)$ as well as by other scholars. Orel (2014) recommends, "future research needs to focus on understanding the implication of differences in race, ethnicity, cultural environments, socioeconomic status and age among LGBT older adults, utilizing the intersectionality perspective that examines multiple identities and the way in which they interact" (p. 70). Scholars Chae and Walters (2009) suggested that the negative influences of discrimination on the health of two-spirit $\mathrm{AI} / \mathrm{NH}$ people calls for additional research on the intersections of both race and sexual orientation (Chae \& Walters, 2009). The current study also supports the need for this type of examination.

Our findings indicate discriminatory actions toward AI/NH people expand beyond health professionals to include encounters throughout their lifetime in the workplace, schools, and in everyday living. This, too, must be addressed given the outcomes that result from it. It is also important to acknowledge that the discrimination our participants talked about are in addition to a long history of violence against $\mathrm{AI} / \mathrm{NH}$ people that began at colonization, with the destruction to their lands, language, culture and communities, and continues today. This violence, hate, and discrimination toward AIs/NHs have resulted in higher rates of posttraumatic stress disorder (PTSD) than their White counterparts along with increased vulnerabilities to a number of health and mental health issues 
(Bassett, Buchwald, \& Mason, 2014, p. 431. Particularly alarming, the Harrison-Quitana, et al. study found that in the national transgender discrimination survey 56\% (565) of AI participants reported having attempt suicide compared to $41 \%$ of all other participants and $1.6 \%$ of the general U.S. population (p. 1). Given the daily negative discriminatory and anti-transgender bias encounters faced by $\mathrm{AI} / \mathrm{NH}$ transgender individuals it is vital that education and training of health professionals and others is given high priority.

Our data extend the previous research findings that voice concerns and highlight the need for specific and unique transgender care. The need for a holistic approach is equally important given that male to female participants should have both the breasts and prostates checked. Participants in this study noted the lack of attention to breast health during medical visits; this is especially concerning given the fact that MTF individuals are found to have higher risk for breast cancer due to hormone therapy. It is recommended that they receive the same screening given to biological women along with screening for prostatic disease and prostate cancer (Roberts \& Fantz, 2014, p. 986).

The transgender specific health care need of access to hormones must be addressed immediately given its health risks to many transgender individuals. Access to hormones was the largest subtheme identified and certainly brings to focus a major health concern noted by many transgender individuals. This study found that cost and lack of insurance was a barrier to obtaining hormones and if individuals could not afford them, they strategically found ways to obtain them even if the hormones were non-prescribed. This study concurs with the findings of a study of transgender people in Ontario, Canada, which found that individuals obtained non-prescribed hormones when they didn't find them available (Rotondi, 2013). It is fairly certain that these types of behaviors are driven by financial and lack of health care service considerations. Clearly, it is evident that more research is needed to understand health impacts and factors that contribute to the use of non-prescribed hormones and that we must find ways to make the hormonal transition safe and affordable or people will continue to put themselves at risk for health complications.

It was encouraging to learn that in May 2013 the Indian Health Service (IHS) National Pharmacy and Therapeutics Committee hosted a discussion on the management of patients treated with hormone therapy for gender dysphoria. Following the protocols created by the Veterans administration, they "determined that the most appropriate estrogen agent for male to female transgender therapy is Estradiol." It is unclear however if all IHS facilities adhere to this recommendation given that one 
participant in this study noted, "IHS on the reservation gives premarin . . . she advocates for them to try to get estradiol because it is healthier."

It is still unclear what the impact, if any, that the Affordable Care Act will have on accessing healthcare, specifically quality hormones. Hopefully, it may provide a means for better access and less risk for this population. What remains evident however, is that transgender individuals will continue to strategize and do what is necessary to access transition related care for it is essential to their basic health and wellbeing.

While participants in this study described the constant negative encounters in their lives, they also spoke of their ability to rebound and recover. This capacity and the ensuing strength should be further explored and utilized as resiliency factors in health interventions if we are to decrease the health disparities. It has been argued that understanding resilience among transgender populations is important for reducing their stress (Bockting et al., 2013), building their strength and strategies to cope with adversity (Singh, et al, 2011), and for practitioners who work with them (Singh \& McKleroy, 2011; Singh, Hays, \& Watson, 2011).

This study supports recent research enumerating how activism, role models, and engagement with other like individuals may contribute to the resiliency and positive health outcomes (Singh, et al., 2011; Testa, Jimenez, \& Rankin, 2014; Singh \& McKleroy, 2011). Unique to the population in the current study was the role that culture and history played in building resiliency. Further research on resilience would have great value and could potentially lead to more effective avenues for intervention and thereby, a reduction in the health disparity gaps for $\mathrm{AI} / \mathrm{NH}$ transgender individuals. However, this author concurs with researchers Luthar, Cicchettie and Becker (2000) that studies for the transgender population must pay careful attention to conceptual and methodological factors for the outcomes to be useful. The population of study must be involved in every aspect of the research that is conducted.

Patient-provide communication with health officials is clearly in a state of disparity for the transgender individuals in this study, excluding some perhaps rare instances where doctors appear helpful and understanding regarding hormone treatment and insurance issues. Besides a need for better patient-provider communication, several other areas of communication seem deficit both with regard to the health needs and wants of $\mathrm{AI} / \mathrm{NH}$ transgender individuals and research programs. Investigating and correcting the stigma of $\mathrm{AI} / \mathrm{NH}$ transgender individuals in the larger population social institutions and media portrayals is an important beginning. Thus, research and educational efforts to the larger non-transgender population is needed as well, beginning with educational programs from K-12 to medical school and beyond, about these various cultures and the intersectionality of these varied cultural identities. Re- 
search to further understand and assist $\mathrm{AI} / \mathrm{NH}$ transgender peoples is critically needed, but so is research on the media and other social institutional hegemony that perpetuates the health disparities addressed in this study.

\section{CONCLUSION}

A major implication of this study focuses on the resiliency of the $\mathrm{AI} / \mathrm{NH}$ transgender population and the factors that contribute to building that resiliency. These findings serve as a starting point for more in depth studies as well as the development of effective health interventions. Building AI/NH resiliency using a cultural foundation will provide additional benefits to aging $\mathrm{AI} / \mathrm{NH}$ transgender people who have expressed loneliness and experience a lack of support and community. If the gaps in health disparities are to be closed, then special consideration must be given to the importance of culture in building resiliency in this population. It is suggested that the 'Indigenist' stress-coping model be used as a conceptual research framework, given its emphasis on cultural strengths (Walters, Simoni, \& Evans-Campbell, 2002). If it is grounded firmly in the CBPR process, the research design, recruitment, development of culturally appropriate measures and assurance of cultural centeredness is ensured. Most importantly, research within this population must consider the environment in which the individuals live for there are layers of influence that impact health and health seeking behavior.

It is acknowledged that this study has some limitations. It was limited in size and gender diversity therefore cannot be generalized to all AI/ $\mathrm{NH}$ transgender individuals. The sample was homogeneous with few female to male transgender individuals. The inclusion of more female to male individuals may have provided different perspectives on the issues under study. Although there are many commonalities found among AI/ $\mathrm{NH}$ populations there are also important differences such as health care systems and sovereign status that may have significant impact, hence it is recommended that researchers who build upon this study do so by examining $\mathrm{AI}$ and $\mathrm{NH}$ populations independently since regional differences were found in the data. Not having a NH in the $\mathrm{CAB}$ reviews presented a bias $\mathrm{CAB}$ committee. This study may have also been strengthened through the development of more questions on impact and influences of culture given its importance on the attitudes, beliefs, and practices of AI/ $\mathrm{NH}$ transgender individuals.

This exploratory study provides valuable insight into the lives, health needs, and health seeking behaviors of transgender AI/NH individuals. It examines commonalities with other transgender populations as well as differences. For this specific population, a major research focus should be on health care access with a closer examination of AI/NH en- 
counters with health professionals, quality of care particularly as it relates to hormones, and the factors that contribute to resiliency. Elder needs should also be examined given the concerns noted. Foremost, AI/ $\mathrm{NH}$ cultures and histories should be studied in the context of the social determinates of health given how they influence life choices, opportunities, and challenges, and ultimately health outcomes and health seeking behaviors.

\section{REFERENCES}

Balsam, K.F., Bu, H., Fieland, K.C, Simoni. J.M., \& Walters, K.L. (2004). Culture, trauma, and wellness: a comparison of heterosexual and lesbian, gay, bisexual and two-spirit Native Americans. Cultural Diversity and Ethnic Minority Psychology, 10(3),287-301.

Barnes, P.M., Adams, P.F., Powell-Griner, E. (2010). Health characteristics of the American Indian or Alaska Native adult population: United States, 2004-2009. National Health Statistics Reports; 20. Hyatttsville, MD: National Center for Health Statistics.

Bassett, D., Buchwald, D., \& Mason, S. (2014). Posttraumatic stress disorder and symptoms among American Indians and Alaska Natives: a review of the literature. Social Psychiatry and Psychiatric Epidemiology, 49,417-433.

Bockting, W.O., Miner, M.H., Swinburne Romine, R.E., Hamilton, A., \& Coleman, E. (2013). Stigma, mental health, and resilience in an online sample of the US transgender population. American Journal of Public Health, e1-e9, online publication, e1-e9. doi:10.2105/ AJPH.2013.301241. Cassels, S., Pearson, C.R., Walters, K., Simoni, J.M., \& Morris, M. (2010). Sexual partner concurrency and sexual risk among gay, lesbian, bisexual, and transgender American Indian/Alaska Natives. Sexually Transmitted Diseases, 37(4),272-278.

Chae, D.H., \& Walters, K.L. (2009). Racial discrimination and racial identity attitudes in relation to self-rated health and physical pain and impairment among two-spirit American Indians/Alaska Natives. American Journal of Public Health, 99(S1), S144-S151.

Charmaz, K. (2006). Constructing grounded theory: a practical guide through qualitative analysis. Los Angeles, CA: Sage Publications.

Clark, M.E., Landers, S., Linde, R., \& Sperber, J. (2001). The GLBT Health Access Project: a state-funded effort to improve access to care. American Journal of Public Health, 91(6),895-896.

Dorsey, R., Graham, G., Glied, S., Meyers, D., Clancy, C., \& Koh, H. (2014). Implementing health reform: improved data collection and 
the monitoring of health disparities. Annual Review of Public Health, 25,123-38.

Ellingson, L., \& Odo, C. (2008). HIV risk behaviors among Mahuwahine (Native Hawaiian transgender women). AIDS Education and Prevention, 20(6),558-569.

Feldman, J., \& Safer, J. (2009). Hormone therapy in adults: suggested revision to the sixth version of the standards of care. International Journal of Transgenderism, 11(3),146-182.

Fredriksen-Goldsen, K.I., Cook-Daniels, L., Kim, H., Erosheva, E.A., Emlet, C.A., Hoy-Ellis, C.P., Goldsen, J., \& Muraco, A. (2014). Physical and mental health of transgender older adults: an at-risk and underserved population. The Gerontologist, 54(3),488-500.

Grant, J.M., Mottet, L.A., \& Tanis, J. (2011). Injustice at Every Turn: A Report of The National Transgender Discrimination Survey. Washington, DC: National Center for Transgender Equality and National Gay and Lesbian Task Force.

Harrison-Quitana, J., Fitzgerald, E, \& Grant J. (2012). Injustice at Every Turn: A look at American Indian and Alaskan Native respondents in the National Transgender discrimination survey. Washington, DC: National Center for Transgender Equality and National Gay and Lesbian Task Force.

Indian Health Services. (June 2013). NPTC formulary brief - gender dysphoria. IHS National Pharmacy and Therapeutics Committee Gender Dysphoria, Gender Identity Disorder. Clinical Guidance. Indian Health Services website. Retrieved January 20, 2016 from https://www.ihs.gov/nptc/clinicalguidance/.

Jones, D.A. (2006). The persistence of American Indian health disparities. American Journal of Public Health, 96(12),2122-2134.

Lombardi, E. (2010). Transgender health: A review and guidance for future research - proceedings from the summer institute at the Center for Research on Health and Sexual Orientation, University of Pittsburgh. International Journal of Transgenderism, 12(4),211-229.

Luthar, S.S., Cicchetti, D., \& Becker, B. (2000). The construct of resilience: a critical evaluation and guidelines for future work. Child Development, 71(3),543-562.

Mayer, K.H., Bradford, J.B., Makadon, H.J., Stall, R., Goldhammer, H., \& Landers, S. (2008). Sexual and gender minority health: what we know and what needs to be done. American Journal of Public Health, 98(6),989-995. 
National Institute of Medicine. (2011). The Health of Lesbian, Gay, Bisexual, and Transgender People: Building a Foundation For Better Understanding. Washington, DC: The National Academies Press.

Newman, S.D., Andrews, J.O., Magwood, G.S., Jenkins, C., Cox, M.J., \& Williamson, D.C. (2011). Community advisory boards in community-based participatory research: a synthesis of best processes. Preventing Chronic Disease, 8(3), A70. Retrieved September 19, 2014, from http://www.cdc.gov/pcd/issues/2011/may/10_0045.htm.

Obedin-Maliver, J., Goldsmith, E.S., Stewart, L., White, W., Tran, E., Brenman, S., Wells, M., Fetterman, D.M., Garcia, G., \& Lunn, M.R. (2011). Lesbian, gay, bisexual, and transgender-related content in undergraduate medical education. Journal of American Medical Association, 306(9),971-977.

Odo C., \& Hawelu, A. (2001). Eo na Mâhû o Hawai'i: the extraordinary health needs of Hawai'i's Mâhû. Pacific Health Dialog, 8(2),327334.

Operario, D., \& Nemoto T. (2005). Sexual risk behavior and substance use among sample of Asian Pacific Islander transgendered women. AIDS Education and Prevention, 17(5),430-443.

Operario, D., Nemoto, T., Iwamoto, M. \& Moore T. (2011). Risk for HIV and unprotected sexual behavior in male primary partners of transgender women. Archives of Sexual Behavior, 40,1255-1261.

Orel, N.A. (2014). Investigating the needs and concerns of lesbian, gay, bisexual, and transgender older adults: the use of qualitative and quantitative methodology. Journal of Homosexuality, 61(1),523-78.

Pachecho, C.M., Daley, S.M., Brown, T., Flippi, M., Greiner, K.A., \& Daley, C.M. (2013). Moving forward: breaking the cycle of mistrust between American Indians and researchers. American Journal of Public Health, 103(12),2152-2159.

Potter, J., Goldhammer, H., \& Makadon, H.J. (2008). Clinicians and the care of sexual minorities. In H.J. Makadon, K.H. Mayer, J. Potter, \& H. Goldhammer (Eds.), Fenway: Guide to Lesbian, Gay, Bisexual, and Transgender Health, 3-24. Philadelphia: American College of Physicians.

Redfern, J.S., \& Sinclair, B. (2014). Improving health care encounters and communication with transgender patients. Journal of Communication in Healthcare, 7(1),25-40.

Roberts, T.K. \& Fantz, C.R. (2014). Barriers to quality health care for transgender population. Clinical Biochemistry, 47,983-987. 
Roscoe, W. (1998). Changing Ones: Third and Fourth Genders in Native North America. New York, NY: St. Martin's Press.

Rotondi, N.K., Bauer, G.R., Scanlon, K., Kaay. M., Travers, R., \& Travers, A. (2013). Nonprescribed hormone use and self-performed surgeries: "do-it-yourself" transition in transgender communities in Ontario, Canada. American Journal of Public Health, 103(10),18301836.

Shaghaghi, A., Bhopat, R.S., \& Sheikh, A. (2011). Approaches to recruiting "hard-to-reach' populations into research: a review of literature. Health Promotion Perspectives, 1(2),1-9.

Shipherd, J.C., Green, K.E., \& Abramovitz. (2010). Transgender clients: Identifying and minimizing barrier to mental health treatment. Journal of Gay \& Lesbian Mental Health, 14,94-108.

Simoni, J.M., Walters, K.L., Balsam, K.F., \& Meyers, S.B. (2006). Victimization, substance use, and HIV risk behaviors among gay/bisexual/2-spirit and heterosexual American Indian men in New York City. American Journal of Public Health, 96(12),2240-2245.

Singh, A.A., Hays, D.G., \& Watson, L.S. (2011). Strength in the face of diversity: resilience strategies of transgender individuals. Journal of Counseling and Development, 89(1),20-27.

Singh, A.A., \& McKleroy, V.S. (2011). "Just getting out of bed is a revolutionary act": the resilience of transgender people of color who have survived traumatic life events. Traumatology, 17(2),34-44.

Snelgrove, J.W., Jasudavisius, A.M., Rowe, B.W., Head, E.M., \& Bauer, G.R. (2012). "Completely out-at-seas" with "two-gender medicine": a qualitative analysis of physician-side barriers to providing healthcare for transgender patients. BMC Health Services Research $12: 110$.

Strickland, J.C. Conducting focus groups cross-culturally: experiences with pacific northwest Indian people. Public Health Nursing, 16(3),190-197.

Testa, R.J., Jimenez, C.L., \& Rankin, S. (2014). Risk and resilience during transgender identity development: the effects of awareness and engagement with other transgender people on affect. Journal of Gay \& Lesbian Mental Health, 18(1),31-46.

Testa, R.J., Sciacca, L.M., Wang, F., Hendricks, M.L., Goldblum, P., Bradford, J., \& Bongar B. (2012). Effects of violence on transgender people, Professional Psychology: Research and Practice 43(5),452-459. 
Thomas, L.R., Rosa, C., Forcehimes, A., \& Donovan, D.M. (2011). Research partnerships between academic institutions and American Indian and Alaska Native tribes and organizations: effective strategies and lessons learned in a multisite CTN study. American Journal of Drug and Alcohol Abuse, 37(5),333-338.

U.S. Commission on Civil Rights. (2004). Broken Promises: Evaluating the Native American Health Care System. Washington, DC: U.S. Commission on Civil Rights.

Vernon, I.S. (2012). HIV/AIDS issues for Native women. In J.R. Joe, \& F.C. Gachupin (Eds.), Health and Sociocultural Issues of Native American Women, 169-190. Connecticut: Praeger Press.

Wallerstein, N., \& Duran, B. (2010). Community-based participatory research contributions to intervention research; the intersection of science and practice to help improve health equity. American Journal of Public Health, Supp1(100),S40-S46.

Walters, K.L., Simoni, J.M., \& Evans-Campbell, T. (2002). Substance use among American Indians and Alaska Natives: incorporating culture in an "Indigenist" stress-coping paradigm. Public Health Reports, 117(S1),S104-S117.

Yuan, N.P., Duran, B.M., Walters, K.L., Pearson, C.R., \& Evans-Campbell, T.A. 2014. Alcohol misuse and associations with childhood maltreatment and out-of-home placement among urban two-spirit American Indian and Alaska Native people. International Journal of Environmental Research and Public Health, 11,10461-1047. 